\title{
Real Time Emulation of COPE-like Network Coding in FANET using ns-3
}

\author{
D. S. Meitis ${ }^{1}$, D. S. Vasiliev ${ }^{2}$, I. Kaysina ${ }^{3}$, A. Abilov ${ }^{4}$, V. Khvorenkov ${ }^{5}$ \\ ${ }^{1}$ Development Bureau, \\ CJSC NPO Telecom, \\ Izhevsk, Russian Federation \\ E-mail: ${ }^{1}$ dsmejtis@npotelecom.ru \\ ${ }^{2-4}$ Department of Communication Networks and Systems, \\ ${ }^{5}$ Department of Radio Engineering, \\ Kalashnikov Izhevsk State Technical University, \\ Izhevsk, Russian Federation \\ E-mail: ${ }^{2}$ danil.s.vasilyev@istu.ru, ${ }^{3}$ irina.kaysina@ istu.ru, ${ }^{4}$ albert.abilov@istu.ru
}

Received: June 14, 2018

\begin{abstract}
In this paper, we consider the version of network coding (COPE-like), that allow compressing data for more effective usage of the wireless medium. We discuss the possibility of its application in the networks of Unmanned Aerial Vehicles (UAV) - Flying Ad hoc Networks. We have carried out an emulation of the network coding usage and analyzed its results.
\end{abstract}

Keywords: network coding, B.A.T.M.A.N, Flying Ad-hoc Networks, Unmanned Aerial Vehicles, ns-3.

\section{INTRODUCTION}

Wireless networks assume using common medium for data transmission between the nodes. Multiple access methods such as TDMA, FDMA and CDMA are used to access the transmission medium. In this case, each node hears all messages in the network but drops ones addressed to the other nodes.

Network coding (NC) is a spectral efficient aid to multicasting [1-7]. COPE-like NC [8] makes it possible to reduce the ineffective use of the transmission medium by compressing two packets transmitted on the network into one. The principle of COPE-like network coding is shown in Figure 1. Node A sends packets to node $\mathrm{C}$ and node $\mathrm{C}$ sends packets to node $\mathrm{A}$. Node B is a NC-enabled relay. Using COPE-like NC we need only 3 timeslots instead of 4 timeslots in traditional approach.

Network coding in this form (COPE-like) can be used in Unmanned Aerial Vehicles (UAV) networks (Flying Ad hoc Networks, FANETs) for efficient data transmission using intermediate nodes (drones) [9-10]. In our previous articles we estimated efficiency of data delivery in FANETs using simulation setup with and without COPE-like Network Coding [11-12]. The following sections consider the emulation of an UAV network of three nodes and analyze the results and prospects of using network coding in them.

(C) Meitis D. S., Vasiliev D. S., Kaysina I., Abilov A., Khvorenkov V. 2018 


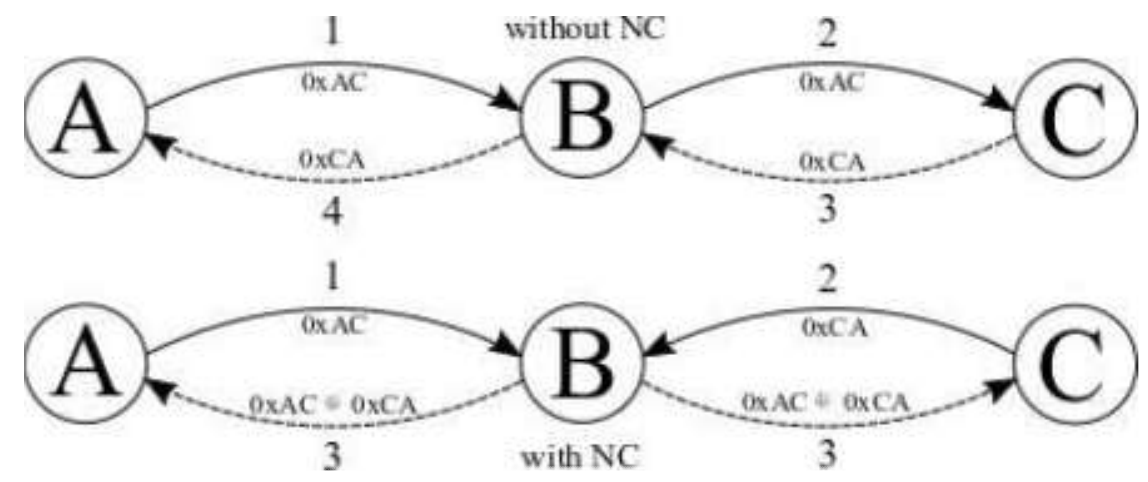

Figure 1. The principle of COPE-like network coding

\section{EMULATION DETAILS}

Network emulation scheme with the Ubuntu (Linux) virtual machines that are physically connected through the discrete-event network simulator for Internet systems called ns-3 was chosen as a testbed for network coding. The advantages of the presented solution are that the use of virtual machines instead of simulated nodes brings results closer to reality, and since the transmission medium itself is a simulation, we can implement any network topology, the movement of nodes in it, the standard of wireless data transmission and the model of transmission errors. Figure 2 shows the scheme and the used stack.

\begin{tabular}{|c|c|c|c|}
\hline $\begin{array}{c}\text { Guest machine A } \\
\text { Ubuntu } 16.04\end{array}$ & $\begin{array}{c}\text { Guest machine B } \\
\text { Ubuntu } 16.04\end{array}$ & $\begin{array}{c}\text { Guest machine C } \\
\text { Ubuntu } 16.04\end{array}$ & \multirow{4}{*}{$\begin{array}{l}\text { Guest machines } \\
\text { in VirtualBox }\end{array}$} \\
\hline iface: enp0s 8 & if ace: enp0s8 & iface: enp $0 \mathrm{~s} 8$ & \\
\hline MAC: FE:00:00:00:0000 & MAC: FE:00;00;00;00:01 & MAC: FE:00:00:00:00:00 & \\
\hline Bridge adapter 2 & Bridge adapter 2 & Bridge adapter 2 & \\
\hline $\begin{array}{l}\text { Network bridge } \\
\text { iface: br0 }\end{array}$ & $\begin{array}{l}\text { Network bridge } \\
\text { iface: brl }\end{array}$ & $\begin{array}{l}\text { Network bridge } \\
\text { iface: br } 2\end{array}$ & \multirow{2}{*}{$\begin{array}{l}\text { Host machine } \\
\text { Ubuntu } 16.04\end{array}$} \\
\hline $\begin{array}{c}\text { TAP interface } \\
\text { iface: } \operatorname{tap} 0 \\
\text { MAC:FE;00:00:00:00;00 }\end{array}$ & $\begin{array}{c}\text { TAP interface } \\
\text { iface: tap I } \\
\text { MAC: } F E ; 00 ; 00 ; 00 ; 00 ; 01\end{array}$ & $\begin{array}{c}\text { TAP interface } \\
\text { iface: } \operatorname{tap} 2 \\
\text { MAC; FE:00:00;00:00;02 }\end{array}$ & \\
\hline TAP bridge & TAP bridge & TAP bridge & \multirow{2}{*}{$\begin{array}{l}\text { Nodes with TAP } \\
\text { in NS-3 }\end{array}$} \\
\hline WifiPhy node 0 & WifiPhy node 1 & WifiPhy node 2 & \\
\hline
\end{tabular}

Figure 2. Emulation scheme

\section{Nodes emulation details}

Nodes in the scheme are implemented as virtual machines running OS Ubuntu 16.04 (Linux) with a pre-installed package B.A.T.M.A.N. [13][14] that contains the network coding algorithm. We used GStreamer package to transmit 60 seconds long video file as RTP traffic between nodes. This protocol provides the ability to determine transmission losses based on packet headers. 


\section{Medium simulation details}

The mentioned virtual machines are physically connected through a simulator ns-3. We used 802.11ac wireless standard, "OFDM Rate 54 Mbps" signal propagation model and Constant Rate Wi-Fi error model as physical layer of the transmission. We also disabled channel layer frame retransmissions to see more realistic picture of losses.

\section{STATIC NoDES SCHEME}

Firstly, we consider the topology of the network with three static nodes located 50 meters apart. It is shown in Figure 3. The communication between nodes $\mathrm{A}$ and $\mathrm{C}$ is carried out through the relay node $\mathrm{B}$. Nodes $\mathrm{A}$ and $\mathrm{C}$ transmit data each other.

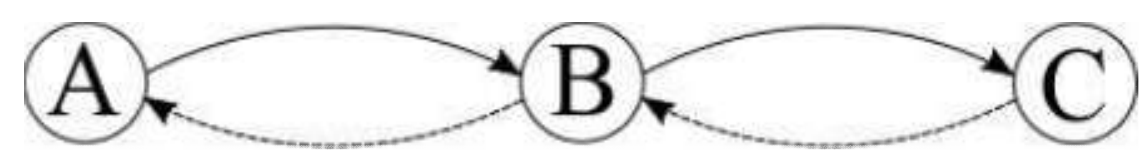

Figure 3. Static nodes scheme

Data transmission was carried out using the network coding algorithm and without it. We tested influence of the bitrate on the transmission quality in this scheme. Data is transmitted on rates 1.25, 2 and 5 Mbps. The results are shown in Figure 4.

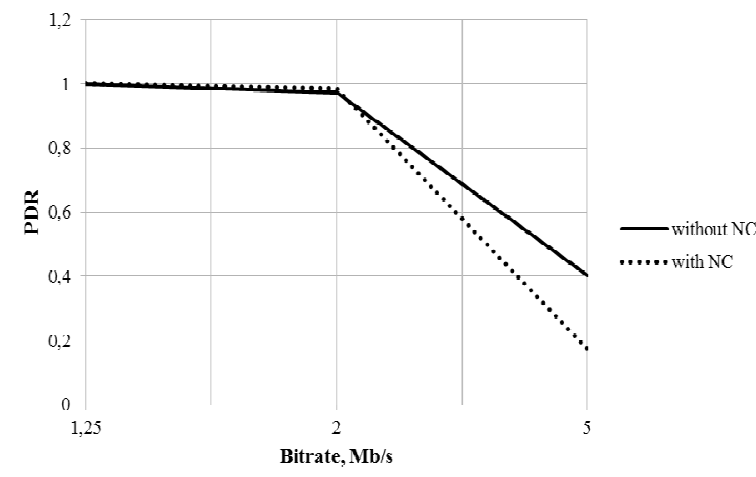

$a$

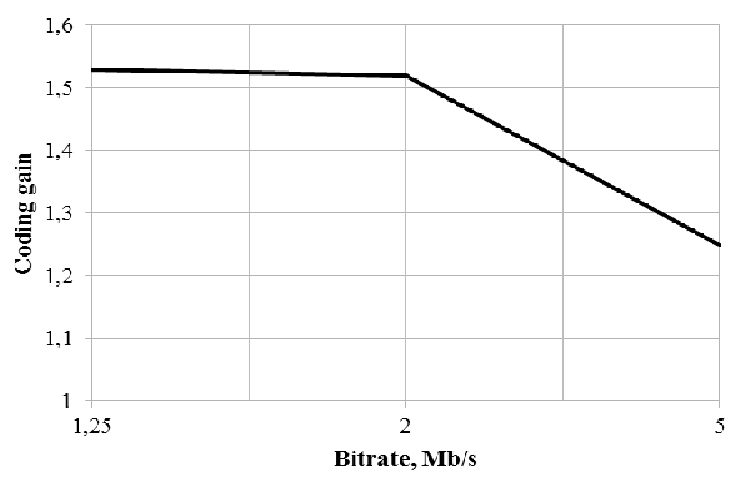

$b$

Figure 4. Results for static relay node scheme:

the dependence of the PDR on bitrate $(a)$; the dependence of the coding gain on bitrate $(b)$

We can estimate the transmission quality by comparing Packet Delivery Ratio (PDR). It is simple metric defined as a ratio of the number of delivered packets to the total number of sent packets:

$$
P D R=\frac{D P}{S P},
$$

where $P D R$ is Packet Delivery Ratio, $D P$ is the count of delivered packets and $S P$ is the total count of sent packets.

On the other hand, [15] introduces the concept of "coding gain" to measure the advantage of network coding. It is the ratio of the number of transmitted packets without applying network coding to the number of packets transmitted in the network using it: 


$$
C G=\frac{S}{S_{n c}}
$$

where $C G$ is coding gain, $S$ is the number of transmitted packets without use of network coding and $S_{n c}$ is the number of transmitted packets using it.

No data loss occurs when data is transmitted at $1.25 \mathrm{Mbps}$. Network coding gives a slight advantage at data delivery with $2 \mathrm{Mbps}$ rate. However, it is inferior to data transfer without the use of network coding at $5 \mathrm{Mbps}$. The degradation of the packet delivery ratio with the high-speed data transmission can be explained by the fact that due to the peculiarities of implementing network coding in the B.A.T.M.A.N., the buffer gets overflowed quickly at the relay node and packets are beginning to be dropped.

Obviously, the higher is coding gain, the more efficient we use the transmission medium. Figure 4 (b) shows a graph of the dependence of the "coding gain" on the bitrate on the relay node. We can see that the relay node uses the transmission medium up to $150 \%$ more efficiently when using network coding.

\section{Mobile Relay Node SCHEME}

Figure 5 shows the second scheme. There are two fixed nodes located at a distance of $\mathrm{X}$ meters from each other, and the relay node that circulates between them at a speed of $35 \mathrm{~m} / \mathrm{s}$, along a circle with a radius of $\mathrm{X} / 4$ meters, centered at the point $\mathrm{X} / 2$ meters.

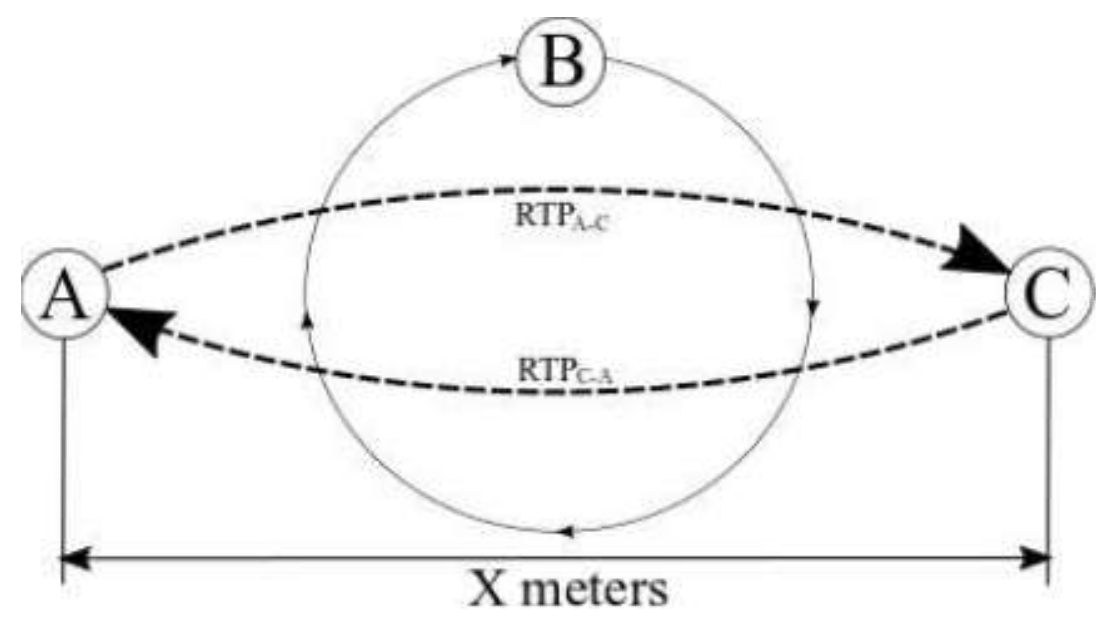

Figure 5. Mobile relay node scheme

As in the first case, video data is transmitted simultaneously from node $\mathrm{A}$ to node $\mathrm{C}$ and backwards. $P D R$ and $C G$ metrics are measured depending on the distance $\mathrm{X}$.

As the distance between nodes and the radius of circulation of the relay node are growing, the quality of data transmission naturally decreases. Particularly nodes A and C are within line of sight at $\mathrm{X}$ equal to 60 meters so the relay node is not used for transmission. And the connection is completely lost with the range of 120 meters.

Since the transmission losses may vary in a high range it would be obviously to measure coding gain relative to the number of delivered packets:

$$
C G_{P D R}=\frac{S}{S_{n c}} \cdot \frac{P D R_{n c}}{P D R}
$$


where $C G_{P D R}$ - is the coding gain relative to the number of delivered packets, $P D R$ is the packet delivery ratio without using coding gain and $P D R_{n c}$ is the packet delivery ratio using coding gain. Figure 6 depicts the dependencies of the $P D R$ and the coding gain relative to $P D R$ from the distance $\mathrm{X}$ between nodes $\mathrm{A}$ and $\mathrm{C}$ using network coding and without it.

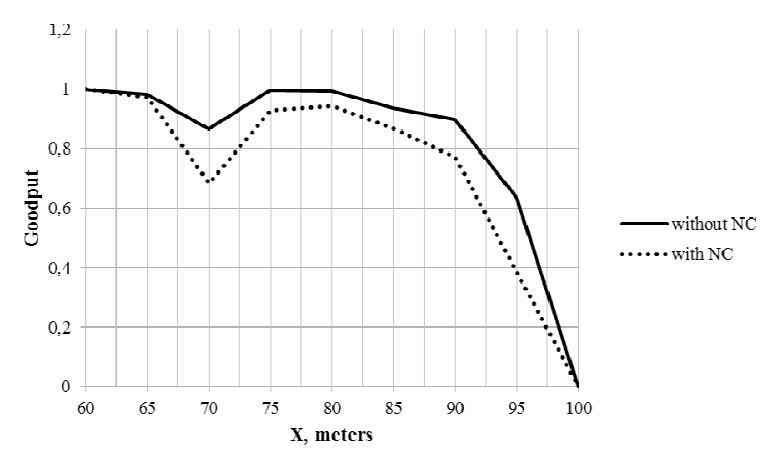

$a$

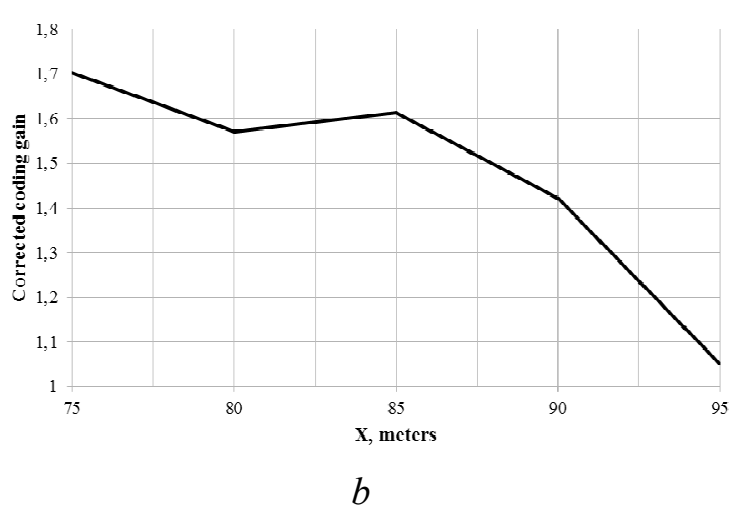

$b$

Figure 6. Results for mobile relay node scheme: $P D R$ - distance dependence $(a) ; C G_{P D R}$ - distance dependence $(b)$

$P D R$ is reduced by an average of $9.5 \%$ with the use of network coding. This can be attributed to the fact that B.A.T.M.A.N. uses long message storage buffers for network coding and the search takes relatively long time therefore the quality of the transmission becomes worse in mobility conditions. Meanwhile Figure 6 (b) shows that the coding gain is also retained for the mobile scheme. The wireless medium is used at 5-70\% more efficiently depending on the distance using network coding. Degrading of the coding gain with the high range between the nodes as well as $P D R$ is associated with increased delays.

\section{CONCLUSION}

According to the results of real-time emulation, we can say that network coding is a powerful tool that allows using the transmission medium resources more efficient. It is especially important in wireless networks. Real-time network emulation with the mobile relay node shows that network coding has the potential for both static topologies and mobile ones. Network coding reduces amount of the transmitted packets in network for 5-70\% depending on transmission reliability. However, we should take into account that the larger message buffers for decoding, the longer delays searching for the desired message. In the future work it is necessary to find a balance between the permissible delays and the efficiency of data compression.

The reported study was funded by RFBR according to the research project № 18-37-00218 mol_a.

\section{REFERENCES}

1. Ahlswede, R., Li, S.-Y., \& Yeung, R. (2000). Network information flow. IEEE Transactions on Information Theory, 46(4), 1204-1216. doi: 10.1109/18.850663.

2. Yeung, R. W. (2010). Network coding theory: An introduction. Frontiers of Electrical and Electronic Engineering in China, 5(3), 363-390. doi: 10.1007/s11460-010-0103-1.

3. Pahlevani, P., Hundeboll, M., Pedersen, M., Lucani, D., Charaf, H., Fitzek, F., Bagheri, H., \& Katz, M. (2014). Novel concepts for device-to-device communication using network coding. IEEE Communications Magazine, 52(4), 32-39. doi: 10.1109/MCOM.2014.6807944. 
4. Vieira, L. F. M., Gerla, M., \& Misra, A. (2013). Fundamental limits on end-to-end throughput of network coding in multi-rate and multicast wireless networks. Computer Networks, 57(17), 3267-3275. doi: 10.1016/j.comnet.2013.07.015.

5. Goseling, J., Gastpar, M., \& Weber, J. H. (2015). Random access with physical layer network coding. IEEE Transactions on Information Theory, 61(7), 3670-3681. doi: 10.1109/TIT.2015.2425879.

6. Chou, P. A., \& Wu, Y. (2007). Network coding for the internet and wireless networks. IEEE Signal Processing Magazine, 24(5), 77-85. doi: 10.1109/MSP.2007.904818.

7. Pyattaev, A., Galinina, O., Andreev, S., Katz, M., \& Koucheryavy, Y. (2015). Understanding practical limitations of network coding for assisted proximate communication. IEEE Journal on Selected Areas in Communications, 33(2), 156-170. doi: 10.1109/JSAC.2014.2384232.

8. Katti, S., Rahul, H., Hu, W., Katabi, D., M'edard, M., \& Crowcroft, J. (2006). XORs in the air: practical wireless network coding. IEEE/ACM Transactions on Networking, 16(3), 497-510. doi: 10.1109/TNET. 2008.923722.

9. Kaysina, I. A., Vasiliev, D. S., \& Abilov, A. V. (2018). Setevoe kodirovanie v setyah FANET [Network coding in FANET]. Electrosvyaz, 2018(1), 64-68 (in Russian).

10. Kaysina, I. A., Vasiliev, D. S., Abilov, A., Meitis, D. S., \& Kaysin, A. E. (2018). Performance evaluation testbed for emerging relaying and coding algorithms in Flying Ad Hoc Networks. In: 2018 Moscow Workshop on Electronic and Networking Technologies (MWENT) (pp. 1-5). Moscow, Russia : IEEE. doi: 10.1109/MWENT.2018.8337169.

11. Vasiliev, D. S., Kaysina, I. A., \& Abilov, A. (2017). Performance evaluation of COPE-like network coding in Flying Ad Hoc Networks: Simulation-based study. In: O. Galinina, S. Andreev, S. Balandin, Y. Koucheryavy (Eds.), Internet of Things, Smart Spaces, and Next Generation Networks and Systems. ruSMART 2017, NsCC 2017, NEW2AN 2017. Lecture Notes in Computer Science, 10531 (pp. 577-586). Cham, Switzerland: Springer. doi: 10.1007/978-3-319-67380-6_54.

12. Vasiliev, D. S., Meitis, D. S., \& Abilov, A. (2014). Simulation-based comparison of AODV, OLSR and HWMP protocols for Flying Ad Hoc Networks. In: S. Balandin, S. Andreev, Y. Koucheryavy (Eds.), Internet of Things, Smart Spaces, and Next Generation Networks and Systems. NEW2AN 2014. Lecture Notes in Computer Science, 8638 (pp. 245-252). Cham, Switzerland : Springer. doi: 10.1007/978-3-319-10353-2_21.

13. Jungic, D. A. (2011). Better Approach to Mobile Ad hoc Networking [Slides]. Retrieved from http://www2. ensc.sfu.ca/ 1jilja/ENSC427/Spring11/Projects/team9/ENSC427_Group9_batman_pres.pdf.

14. Neumann, A., Aichele, C., Lindner, M., \& Wunderlich, S. (2008). Better approach to mobile ad-hoc networking (B.A.T.M.A.N.) IETF draft (pp. 1-24). Retrieved from https://www.researchgate.net/publication/ 320172464_Better_approach_to_mobile_ad-hoc_networking_BATMAN.

15. Ngai, C. K., \& Yeung, R. W. (2004). Network coding gain of combination networks. In: Information Theory Workshop (pp. 283-287). San Antonio, USA : IEEE. doi: 10.1109/ITW.2004.1405315. 